\title{
Successful Birth in a Case of Cervical Cancer Following Total Laparoscopic Nerve Sparing Radical Trachelectomy with Sentinel Node Dissection and Preventive Cerclage
}

\author{
Marie CV Ramilo Mendoza ${ }^{1}$, Kuan-Gen Huang ${ }^{2}$
}

\begin{abstract}
Aim and objective: The aim of this case report is to show the feasibility of a fertility-sparing and nerve-sparing procedure for early-stage cervical cancer to preserve the patient's reproductive ability and minimize the surgical complications associated with the radical surgery while providing adequate treatment to ensure good oncologic outcome.

Background: The standard management for early-stage cervical cancer is radical hysterectomy with pelvic lymphadenectomy or concurrent chemoradiation. Both are associated with good survival, but neither form of therapy allows the preservation of a functional utero-ovarian system for future fertility.

Case description: We present a young woman with cervical adenocarcinoma stage IB1 who underwent laparoscopic nerve-sparing radical trachelectomy with sentinel node dissection and preventive cerclage. There were no intraoperative or postoperative complications. The patient had a successful pregnancy 2 years after the operation and no evidence of disease for more than 5 years.

Conclusion: With laparoscopic nerve-sparing radical trachelectomy, a woman's reproductive ability is maintained while ensuring good oncologic survival. It is also associated with less surgical and postoperative morbidity, such as urinary and sexual dysfunction, which are common in the traditional technique of radical trachelectomy.

Clinical significance: This technique of radical trachelectomy provides the chance to retain future fertility for possible conception while minimizing patient morbidity and ensuring good prognosis.

Keywords: Cerclage, Cervical cancer, Fertility-sparing surgery, Lymphadenectomy, Radical trachelectomy.

Journal of South Asian Federation of Obstetrics and Gynaecology (2020): 10.5005/jp-journals-10006-1808
\end{abstract}

\section{BACKGROUND}

Early-stage cervical cancer patients have been traditionally treated with radical hysterectomy and pelvic lymph node dissection resulting in long-term complications, such as urinary dysfunction, sexual dysfunction, and loss of fertility. ${ }^{1,2}$ Since young women have become disproportionately affected by cervical cancer over the years, the desire to retain their reproductive ability has become an important concern for this age-group. Radical trachelectomy is an alternative for these women who wish to preserve future fertility. Furthermore, nerve-sparing efforts have been incorporated to address the need to minimize postoperative morbidity such as bladder dysfunction. The surgical technique and approach continue to evolve, as it may be done vaginally, abdominally, or utilizing a minimally invasive approach either by conventional laparoscopy or by robotic surgery. The minimally invasive approach is preferred, since it is associated with less complications, shorter hospital stay, and faster recovery. The oncologic outcomes are favorable with the use of fertility-sparing procedures for early-stage cervical disease.

We present a case of total laparoscopic nerve-sparing radical trachelectomy, sentinel node dissection, and preventive cerclage in a young woman who had a successful pregnancy after completing her oncologic treatment. The combination of the minimally invasive approach and the synergy of the procedures contributed to the improved oncologic outcome and reproductive outcome of the patient. We aim to show that this fertility-sparing and nerve-sparing procedure is a viable option for young women with early-stage cervical cancer for preservation of reproductive ability without compromising survival.
${ }^{1}$ Department of Obstetrics and Gynecology, Philippine General Hospital, Manila, Metro Manila, Philippines

${ }^{2}$ Department of Obstetrics and Gynecology, Chang Gung Memorial Hospital, Linkou, Taoyuan, Taiwan-Republic of China

Corresponding Author: Kuan-Gen Huang, Department of Obstetrics and Gynecology, Chang Gung Memorial Hospital, Linkou, Taoyuan, Taiwan-Replublic of China, Phone: +886-3-3281200, e-mail: kghuang@ ms57.hinet.net

How to cite this article: Ramilo Mendoza MCV, Huang K-G. Successful Birth in a Case of Cervical Cancer Following Total Laparoscopic Nerve Sparing Radical Trachelectomy with Sentinel Node Dissection and Preventive Cerclage. J South Asian Feder Obst Gynae 2020;12(4): 261-264.

Source of support: Nil

Conflict of interest: None

\section{Case Description}

A 36-year-old Gravida 2 Para 1 (1011) presenting with postcoital vaginal bleeding and vaginal discharge was found to have an exophytic cervical mass at the anterior lip measuring $1.5 \mathrm{~cm}$ (Fig. 1). Cervical biopsy revealed adenocarcinoma. Complete preoperative evaluation and metastatic investigation were performed. Chest $X$-ray showed no evidence of metastasis. On pelvic magnetic resonance imaging (MRI), the cervical mass was located in the anterior cervical lip measuring $10 \mathrm{~mm}$ in greatest dimension with full thickness involvement of the cervical tip with no uterine, vaginal,

(c) The Author(s). 2020 Open Access This article is distributed under the terms of the Creative Commons Attribution 4.0 International License (https://creativecommons. org/licenses/by-nc/4.0/), which permits unrestricted use, distribution, and non-commercial reproduction in any medium, provided you give appropriate credit to the original author(s) and the source, provide a link to the Creative Commons license, and indicate if changes were made. The Creative Commons Public Domain Dedication waiver (http://creativecommons.org/publicdomain/zero/1.0/) applies to the data made available in this article, unless otherwise stated. 


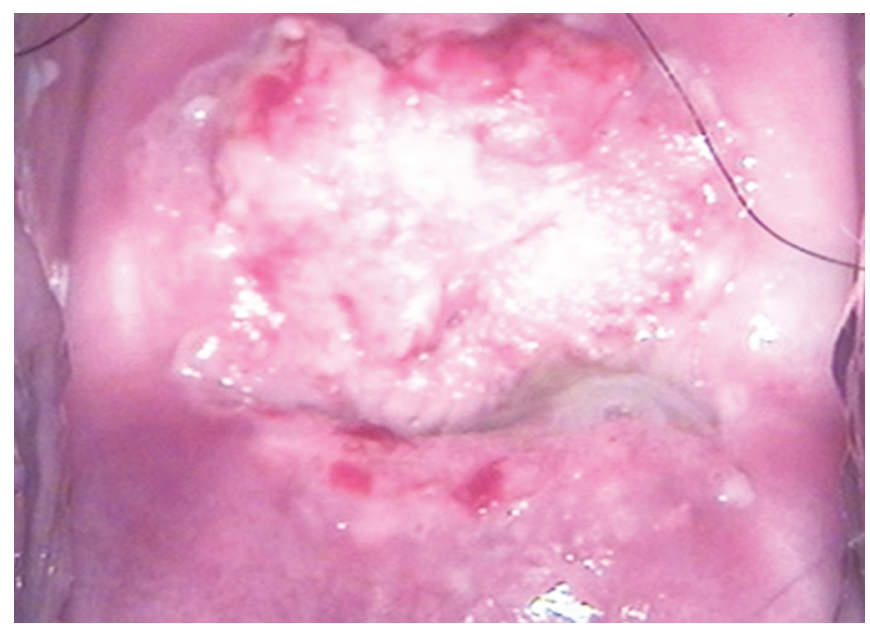

Fig. 1: Cervical mass at the anterior lip of the cervix. Patient's cervical mass examined under colposcopy. The cervical mass exophytic and located at the anterior cervical lip measuring $1.5 \mathrm{~cm}$

parametrial, or end cervical extension. There were no enlarged lymph nodes or other sites of abdominal metastasis. There were two subserous myoma measuring $7 \mathrm{~cm}$ at the left cornu and $2 \mathrm{~cm}$ at the anterior midcorpus. The clinical stage at presentation was stage IB1 (2009 FIGO classification system). With a strong desire for future fertility, the patient decided to undergo radical trachelectomy. A comprehensive preoperative discussion about the procedure and its oncologic and fertility outcomes commenced, and a written informed consent was obtained.

\section{Surgical Technique}

The laparoscopic nerve-sparing radical trachelectomy procedure is a modification of the type $\mathrm{C} 1$ radical hysterectomy with level 1 lymphadenectomy described by Querleu \& Morrow (Q-M classification). ${ }^{3}$ The primary trocar was placed through the LeeHuang point, ${ }^{4}$ and four lateral ancillary ports were inserted. The uterus had smooth serosal surface and was asymmetrically enlarged with two subserous myoma. Both adnexa were grossly normal. Myomectomy was performed. For the sentinel lymph node mapping, diluted patent blue solution was injected at the cervix at the 2-, 5-, 8-, and 11-o'clock positions. Frozen section of the sentinel node along the right pelvic lymph channel was negative for malignancy. En bloc bilateral pelvic lymphadenectomy was done as previously reported. ${ }^{5}$

Since the lymph nodes were negative, the procedure continued with nerve sparing radical trachelectomy. The paravesical and pararectal spaces were developed. The bilateral ureters were mobilized completely from the medial sheath of the broad ligament up to where it entered the lateral parametrial tissue. The uterine artery was traced from its origin from the internal iliac artery, and the descending branch was selectively ligated while preserving the ascending branch of the uterine artery. The hypogastric nerve underneath the ureter and uterosacral ligament, the pelvic splanchnic nerve at the cardinal ligament, and the vesical branches of the inferior hypogastric plexus at the posterior vesicouterine ligament were identified and preserved on both sides. Hysteroscopy was performed to mark the border of carcinomatous area. The cervix was transected below the level of the uterine isthmus to obtain at least $10 \mathrm{~mm}$ tumor-free endocervical margin. The vaginal wall was incised circumferentially to obtain a tumor-free vaginal cuff

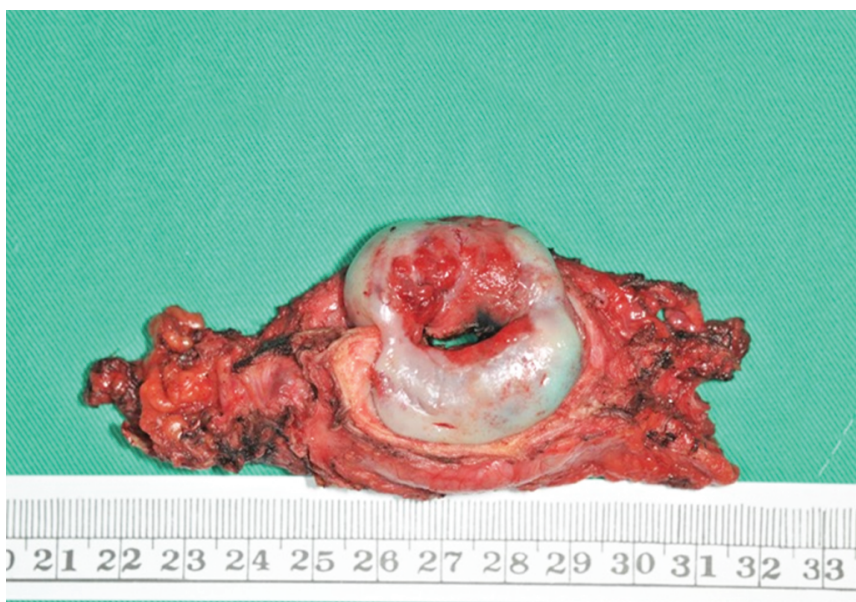

Fig. 2: Excised cervix with vagina and parametrium status post-radical trachelectomy. Postoperative radical trachelectomy specimen showing the entire cervix with vaginal cuff and bilateral parametrium. The cervical mass is located at the anterior lip and as part of radical trachelectomy, a 2-centimeter vaginal margin and all parametrial tissues are included in the specimen

margin of about $2-3 \mathrm{~cm}$. The specimen was removed en bloc with the cervix, vagina, and adequate parametrium (Fig. 2).

The vagino-isthmic approximation was initially performed by laparoscopy to close the margins and continued vaginally using V-Loc ${ }^{\text {TM }}$ (Covidien, Mansfield, MA) 1-0 with a continuous running suture. Abdominal cerclage was carried out laparoscopically by circumferentially placing a Mersilene tape 1-0 around the lower uterine segment. The Mersilene tape was secured posterior to the uterus. Estimated blood loss was $100 \mathrm{~mL}$. There were no postoperative complications.

The final histopathology report revealed well-differentiated adenocarcinoma consistent with stage IB1 with a 2-cm tumor-free vaginal margin. The harvested bilateral pelvic lymph nodes (ten on each side) were negative for malignancy.

\section{Reproductive and Oncologic Outcome}

The patient had an uneventful postoperative follow-up. The neocervix exhibited good healing 4 months after the operation (Fig. 3). The patient had resumption of regular menstrual cycle and had no complaints of vaginal discharge. After undergoing one cycle of in vitro fertilization with negative result, she conceived spontaneously 2 years after the surgery and was able to carry the fetus up to 33 weeks age of gestation. She delivered a live baby girl weighing $2000 \mathrm{~g}$ via classical cesarean section. She has been on regular follow-up since the surgery and has no evidence of disease recurrence for more than 5 years.

\section{Discussion}

The cornerstone of surgical management for early-stage cervical cancer is radical hysterectomy. For patients who are unfit for surgery, the recommended treatment is concurrent chemoradiation. Neither form of therapy allows for the preservation of a functional utero-ovarian system for reproductive purposes. As the number of younger women afflicted with malignancy increases, and as the age for conception becomes more delayed, there is a need to provide treatments that can give the same curative outcome but 


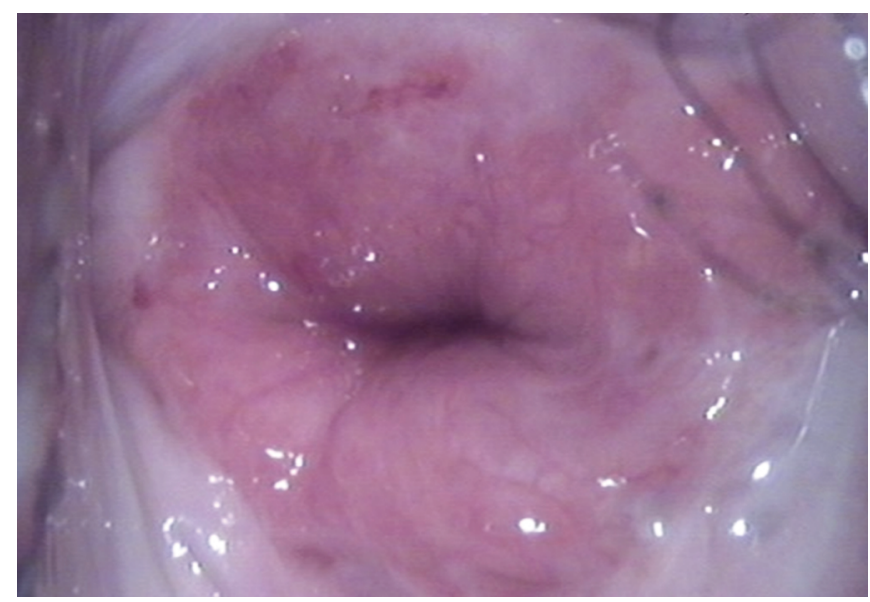

Fig. 3: Neocervix 4 months after surgery. Cervix examined under colposcopy 4 months after the surgery. The new cervix had healed properly and has no signs of infection or erosion

with less associated morbidity for patients with malignancy. Fertility preservation and reproductive health needs have become a main concern for reproductive-age cancer survivors.

The management of early-stage cervical cancer continues to evolve with numerous options differing in the radicality of the procedure and surgical technique. Radical trachelectomy is a fertility-sparing option for a select number of patients. Criteria for this conservative management include diagnosis with a stage IA2 or IB1 tumor, histology of squamous carcinoma, adenocarcinoma, or adenosquamous carcinoma, tumor size less than or equal to 2 $\mathrm{cm}$, no lymphovascular space invasion, and no nodal involvement. ${ }^{6}$ The patient had a thorough preoperative assessment pointing to a stage IB1 that was proven intraoperatively and in the final pathology result. Strict patient selection must be employed to ensure the best treatment option that will balance good oncologic outcome and preservation of reproductive ability is given to the patient.

The concept of radical trachelectomy is the removal of the cervix with adequate vagina and parametrium while preserving the uterine corpus. Total laparoscopic nerve-sparing trachelectomy allows the procedure to be performed under the magnified view of laparoscopy while preserving the autonomic nerve fibers associated with maintenance of good bladder and sexual function. The nervesparing technique was first applied to radical hysterectomy to improve surgical morbidity and lessen neurologic problems. ${ }^{7,8}$ This technique may also be directed to radical trachelectomy, especially since it is often applied to young women who may develop more concerns with regard to their urinary and sexual health needs. In our patient, careful identification and dissection of the hypogastric nerve, pelvic splanchnic nerve, and vesical branches of the inferior hypogastric plexus were performed. The patient did not suffer from any form of bladder dysfunction. The procedure provided the patient the chance to maintain her reproductive ability. Although radical trachelectomy has been associated with early pregnancy loss and preterm birth, the pregnancy rates are high. ${ }^{9}$ Performance of cerclage aids in the prevention of preterm birth.

The role of pelvic lymphadenectomy in cervical cancer is to determine metastasis for both prognostic and therapeutic planning. It has no therapeutic effect; therefore, concerns about the possible sequelae and complications of a complete lymphadenectomy, such as lymphedema, lymphocyst formation, hemorrhage, and nerve injury continue to arise. Sentinel node mapping allows for the determination of the presence or absence of metastasis to the lymph nodes. If the sentinel lymph node is negative, the rest of the lymph nodes will be free of disease precluding the need for a complete lymphadenectomy. Laparoscopic sentinel lymph node mapping contributes to decreased operative morbidity and facilitates complete removal of biologically relevant nodes. ${ }^{10}$

\section{Conclusion}

Laparoscopic nerve-sparing radical trachelectomy is a safe and viable option for young women with early-stage cervical cancer and a strong desire to preserve fertility. It is associated with less surgical and postoperative morbidity, good oncologic survival, and maintenance of reproductive ability.

\section{Clinical Significance}

Nerve-sparing radical trachelectomy provides the chance to retain future fertility for possible conception while minimizing patient morbidity and ensuring good prognosis. This type of surgery can be offered to young women with early-stage cervical cancer with a strong desire for future fertility.

\section{ACKNOWLedgments}

We thank the Asia-Pacific Association for Gynecologic Endoscopy and Minimally Invasive Therapy (APAGE) for providing the International Fellowship in Minimally Invasive Gynecologic Surgery at Chang Gung Memorial Hospital for Dr Mendoza.

\section{Consent}

Written informed consent was obtained from the patient for publication of the case report and accompanying images.

\section{Institutional Review Board Approval}

The Hospital Institutional Review Board ruled that approval was not required for this study.

\section{References}

1. Brooks RA, Wright JD, Powell MA, et al. Long-term assessment of bladder and bowel dysfunction after radical hysterectomy. Gynecol Oncol 2009;114(1):75-79. DOI: 10.1016/j.ygyno.2009.03.036.

2. Landoni F, Colombo A, Milani R, et al. Randomized study between radical surgery and radiotherapy for the treatment of stage IB-IIA cervical cancer: 20-year update. J Gynecol Oncol 2017;28(3):e34. DOI: 10.3802/jgo.2017.28.e34.

3. Querleu D, Morrow CP. Classification of radical hysterectomy. Lancet Oncology 2008;9(3):297-300. DOI: 10.1016/S1470-2045(08)70074-3.

4. Lee C-L, Huang K-G, Jain S, et al. A new portal for gynecologic laparoscopy. J Am Assoc Gynecol Laparoscop 2001;8(1):147-150. DOI: 10.1016/S1074-3804(05)60565-8.

5. Lee C-L, Huang K-G, Wang C-J, et al. Laparoscopic radical trachelectomy for stage Ib1 cervical cancer. J Am Assoc Gynecol Laparosc 2003;10(1):111-115. DOI: 10.1016/S1074-3804(05)60244-7.

6. Ramirez PT, Pareja R, Rendon GJ, et al. Management of low-risk earlystage cervical cancer: should conization, simple trachelectomy, or simple hysterectomy replace radical surgery as the new standard of care? Gynecol Oncol 2014;132(1):254-259. DOI: 10.1016/j. ygyno.2013.09.004.

7. Possover M. Technical modification of the nerve-sparing laparoscopyassisted vaginal radical hysterectomy type 3 for better reproducibility of this procedure. Gynecol Oncol 2003;90(2):245-247. DOI: 10.1016/ S0090-8258(03)00255-5. 
8. Fujii S, Takakura K, Matsumura N, et al. Precise anatomy of the vesico-uterine ligament for radical hysterectomy. Gynecol Oncol 2007;104(1):186-191. DOI: 10.1016/j.ygyno.2006. 07.041.

9. Lu Q, Liu C, Zhang Z. Total laparoscopic radical trachelectomy in the treatment of early-stage cervical cancer: review of technique and outcomes. Curr Opin Obstet Gynecol 2014;26(4):302-307. DOI: 10.1097/GCO.0000000000000081.

10. Fader AN, Edwards RP, Cost M, et al. Sentinel lymph node biopsy in early-stage cervical cancer: utility of intraoperative versus postoperative assessment. Gynecol Oncol 2008;111(1):13-17. DOI: 10.1016/j.ygyno.2008.06.009. 